\title{
A Study of Intrahepatic Cholestasis of Pregnancy and Its Perinatal Outcome in a Tertiary Care Centre
}

\author{
Aishwarya Regunathan ${ }^{1}$, Bhuvana Srinivasan ${ }^{2}$ \\ 1,2 Department of Obstetrics and Gynaecology, Sri Ramachandra Institute of Higher Education \\ and Research, Chennai, Tamilnadu, India.
}

\section{ABSTRACT}

\section{BACKGROUND}

Intrahepatic cholestasis is one of the unique conditions specific to pregnancy and should be kept in mind when a patient presents with excessive pruritus seen during the late second and early third trimester of pregnancy, causing subtle alterations in liver functions with increased serum bile acids. This study intended to determine the prevalence of obstetric cholestasis in our hospital, its course and pregnancy outcome in these women.

\section{METHODS}

This is a prospective cross sectional study, carried out among the women attending the Department of OBG, Sri Ramachandra medical college. A total of 22 cases were observed to have obstetric cholestasis from those who attended the hospital during the study period. The data about the variables like patient identification details, demographic data, symptoms, laboratory parameters, feto-maternal outcomes were recorded like pre-existing medical conditions, mode of delivery, birth weight, Apgar score at $1 \mathrm{~min}$ and $5 \mathrm{mins}$ and admission to NICU were recorded. Data collected were analysed and the data variables were represented appropriately.

\section{RESULTS}

A total of 1813 deliveries were conducted in the study setting during the study period. $1.2 \%$ of cases were observed to have intrahepatic cholestasis. The mean gestational age of onset of obstetric cholestasis was 28 weeks. The mean age of the women considered for the study was $28.5 \pm 2.8$ years $(22-34$ years). The mean serum bile acid observed at the time of admission was $33.6 \pm 25.2$. The mean gestational age at the delivery of the study subjects was $36 \mathrm{~W} \pm 5$ days. $50 \%$ of the women had laboured at term and $50 \%$ of the women had preterm labour. 9 women (41\%) delivered through NVD and 13 women (59\%) delivered through lower segment caesarean section. One patient had recurrent cholestasis. The average Apgar score at 1 minute was 7 and at the end of 5 minutes it was $8.50 \%$ of the children admitted to NICU and 4 women gave the history of admissions into NICU of the babies born even in the previous deliveries, due to varied reasons. No perinatal or maternal mortality was observed during the study period.

\section{CONCLUSIONS}

Intrahepatic cholestasis of pregnancy is common among pregnant women which has an impact on feto-maternal outcomes. It is found to be responsible for a large number of perinatal and neonatal deaths especially after 36 weeks of gestation, but if appropriate antenatal care and timely intervention are implemented outcomes of pregnancy would be better.

\section{KEY WORDS}

Intra hepatic Cholestasis, Pregnancy, Ursodeoxycholic Acid, Pruritus.
Corresponding Author: Dr. Aishwarya Regunathan, Department of Obstetrics and Gynaecology, Sri Ramachandra Institute of Higher Education and Research, Chennai, Tamilnadu, India.

E-mail: draishukrishi@gmail.com

DOI: 10.14260/jemds/2021/789

How to Cite This Article:

Regunathan A, Srinivasan B. A study of intrahepatic cholestasis of pregnancy and its perinatal outcome in a tertiary care centre. I Evolution Med Dent Sci 2021;10(45):3906-3909, 10.14260/jemds/2021/789

Submission 08-11-2021, Peer Review 16-12-2021, Acceptance 22-12-2021, Published 28-12-2021.

Copyright (C) 2021 Aishwarya Regunathan et al. This is an open access article distributed under Creative Commons Attribution License [Attribution 4.0 International (CC BY 4.0)] 


\section{BACKGROUND}

Intrahepatic cholestasis is one of the unique conditions specific to pregnancy and should be kept in mind when the patient presents with excessive pruritus seen during the late second and early third trimester of pregnancy, causing subtle alterations in liver functions with increased serum bile acids. Cholestasis is the most common liver disorder seen during the late second and early third trimester of pregnancy, which is known as obstetric cholestasis (OC) or intrahepatic cholestasis of pregnancy (ICP). ${ }^{1}$

The most common symptom of presentation and a key feature for diagnosis is excessive pruritus typically in the palms and soles, which classically starts in and around 27 weeks of gestation ${ }^{2}$ and seldom occurs before 25 weeks of gestation, ${ }^{3}$ along with abnormal liver function tests and increased levels of serum bile acids, which is usually more than $14 \mathrm{mmol} / \mathrm{L} 1$. Obstetric cholestasis is excluded only if an abnormality is consistent with clinical findings e.g. gallstones on liver ultrasound must be causing biliary obstruction; acute infection with hepatitis $\mathrm{C}$ or hepatitis A on serology. In those women where imaging of the liver is not possible before delivery, scanning is done postnatally if the liver function fails to resolve. ${ }^{4}$

Once identified women were interviewed weekly regarding the nature and severity of their pruritus and any associated features (i.e. malaise, anorexia, dark urine, pale stool, right upper quadrant pain, dry cough and urinary tract infection). ${ }^{4}$ The conditions that are usually accompanied by intrahepatic cholestasis of pregnancy usually resolve after delivery, but generally recur during the subsequent pregnancies. ${ }^{5}$

During pregnancy, the affected cases have increased perinatal morbidity and mortality. The usual complication seen due to obstetric cholestasis is increased risk of adverse obstetrical outcomes, which include stillbirth, respiratory distress syndrome, meconium passage, and foetal asphyxia, 4 as well as a compromise in the maternal quality of life due to sleep deprivation and intense pruritus during the antenatal period and PPH intranatally. ${ }^{6}$

This condition usually has an improved outcome on active management including timely antenatal cardiotocography (CTG) monitoring and elective delivery by 38 weeks of gestation. $^{2}$

There is a wide variation of the etiological factors and pregnancy outcomes as supported by various studies. Because of this, the present study was intended to determine the incidence of OC in our hospital, studying the course of pregnancy and evaluating the pregnancy outcomes in these women.

\section{METHODS}

This was a descriptive cross-sectional study, carried out at the Department of OBG, Sri Ramachandra medical college, among the women attending the antenatal ward to undergo safe delivery, from 01-01-2020 to 31-12-2020. Ethics committee approval was not required hence was not obtained. Informed consent was taken from the study participants before enrolment into the study and they were assured that the clinical management and outcomes were not based upon the study, but depended upon the clinical condition and the discretion of the treating obstetrician in the best interest of the antenatal mother.

The cases were evaluated in detail and all the necessary details of the study variables were collected in pre-designed semi-structured proformas, made to meet the objectives of the study. All the cases were followed in a standardized manner from the time of admission to the discharge of the study participant.

The data about the variables like patient identification details, demographic data, symptoms, laboratory parameters were recorded. All the data about the feto-maternal outcomes like pre-existing medical conditions, mode of delivery, gender of the child, Apgar score at $1 \mathrm{~min}$ and 5 mins and admission to NICU and the final condition of the mother and baby were recorded. The data thus collected was collated into MS Excel worksheets periodically and the patient summary was described in mean and standard deviations for continuous variables and proportions and percentages for discrete variables.

\section{Statistical Analysis}

Data tabulation and analysis were done using Microsoft excel 2013 and SPSS 16. Descriptive statistics which included mean and SD for quantitative variables and frequency and percentages for qualitative variables were calculated.

\section{RESULTS}

The case notes discussed here are the compiled histories of 22 cases, which were having obstetric cholestasis and gave birth in the study setting. During the study period, a total of 1813 deliveries occurred at the study setting and of these 22 cases were found to have obstetric cholestasis and all of them were recruited for the study.

The mean age of the women considered for the study was $28.5 \pm 2.8$ years (22-34 years). Of these, 7 (31.8\%) were primipara, $15(68.2 \%)$ were multipara and only one case had a previous history of OC. The mean serum bile acids observed at the time of admission was $33.6 \pm 25.2$ (2.3-125) The mean gestational age of the study subjects was $36 \mathrm{~W} \pm 5$ days ( $31 \mathrm{~W} \pm 5$ days to 39 weeks ). These women were diagnosed to have OC at the gestational age of around 28 weeks. The majority of women complained of pruritus, especially in the palms and soles.

$50 \%$ of the women had labour at term and $50 \%$ of the women had preterm labour. 9 women (41\%) delivered through NVD and 13 women (59\%) delivered through LSCS, of them 3 were elective and 10 were emergency LSCS. Of the elective cases, 2 were posted as they were cases of previous LSCS, and one case was posted because of complicated dengue and thalassemia. 4 cases had foetal distress and because of that indication, they were posted for LSCS. In one case induction of labour was done in view of OC and in one case induction was done as the AC was $>95$ th percentile, and upon the failed attempt an emergency LSCS was done. The other complications observed were placenta accreta and HELLP syndrome in one each. There were two cases of GDM and one case of hypothyroidism complicated pregnancies. 
Of the babies born, 9 (41\%) were girls and 13 (59\%) were boys. The average birth weight of the babies born was $2.6 \pm 0.8 \mathrm{kgs}(1.15-3.7 \mathrm{kgs})$. The average Apgar score at 1 minute was 7 and at the end of 5 minutes it was $8.50 \%$ of the children admitted to ICU and 4 women gave a history of admissions into NICU of the babies born even in the previous deliveries, due to varied reasons. 9 (41\%) cases had small vessel disease (SVD) and none had arterial vascular disease (AVD).

There was no incidence of stillbirths.

The majority of the cases were managed conservatively by using ursodeoxycholic acid $300 \mathrm{mg}, 2$ (9\%) cases received $150 \mathrm{mg}$. In one case of recurrent OC, the dosage was increased to ursodeoxycholic acid $450 \mathrm{mg}$ in later stages of pregnancy. The cases which were complicated by diabetes received insulin alongside and the case of anaemia received iron infusion.

\begin{tabular}{|ccc|}
\hline Birth Weight & Number of Cases (N=22) & Percentage \\
Less than $2 \mathrm{~kg}$ & 2 & $0.9 \%$ \\
$2-2.5 \mathrm{~kg}$ & 5 & $22.7 \%$ \\
$2.6-3 \mathrm{~kg}$ & 8 & $36.3 \%$ \\
More than $3 \mathrm{~kg}$ & 7 & $31.8 \%$ \\
Gestational age at delivery & Number of cases $(\mathrm{n}=22)$ & Percentage \\
Less than 34 weeks & 2 & $0.9 \%$ \\
34 weeks to 35 weeks +6 days & 3 & $13.6 \%$ \\
36 weeks to 37 weeks & 6 & $27.2 \%$ \\
More than 37 weeks & 11 & $50 \%$ \\
Serum bile acids & Number of cases $(\mathrm{n}=22)$ & Percentage \\
Less than $15 \mathrm{mmol} / \mathrm{L}$ & 6 & $27.2 \%$ \\
15.1- 30 mmol/L & 7 & $31.8 \%$ \\
30.1 $-50 \mathrm{mmol} / \mathrm{L}$ & 6 & $27.2 \%$ \\
More than $50 \mathrm{mmol} / \mathrm{L}$ & 3 & $13.6 \%$ \\
\hline & Table 1 & \\
\hline
\end{tabular}

\section{DISCUSSION}

The mean age of the women was $28.5 \pm 2.8$ years and this finding agreed with the observations made by Padmaja et al., 6 Cardoso PM et al. ${ }^{6}$

The primary finding was excessive pruritus in the palms and soles in and around 28 weeks of gestation and the mean serum bilirubin was $33.6 \pm 25.2$, this finding was similar to the findings made by Padmaja et al.,7 Cardoso PM et al. ${ }^{5}$ Majority of the cases were those treated with ursodeoxycholic acid $300 \mathrm{mg}$ which was in concordance with many other studies along with other routine medications given as a part of antenatal care and to address pregnancyrelated complications.

Prevalence of OC was observed to be $1.2 \%$, whereas it was $1.15 \%$ in the study done by Ghimirie SP et al. ${ }^{8}$ in Nepal, $1.15 \%$ in a study done by Cordoso PM et al. ${ }^{5}$ in Goa, it was 8.2 $\%$ in the study conducted by Padmaja et al. ${ }^{6}$ in India. But recurrent $\mathrm{OC}$ was found to be very much lower in comparison with the other study findings. In our study, $41 \%$ of the women had full term normal vaginal delivery (FTNVD) and $59 \%$ had LSCS, of them $3 / 13$ were elective caesarean sections. $50 \%$ of the women had full-term deliveries, whereas $50 \%$ had preterm deliveries, which was in comparison with the levels observed in Padmaja et al. ${ }^{7}$ study, but was way higher than the standard findings. This is due to the protocol employed to induce the labour at the earliest possible to decrease the adverse outcomes.

The mean gestational age at delivery was around 38 weeks in liaison to the protocol, followed by Keynon et al. ${ }^{2}$ and the deliveries were induced in majority of the cases as the liver enzymes were not improved even on treatment with ursodeoxycholic acid and a similar approach was followed in studies done by Ghimirie SP et al. ${ }^{8}$ but many cases were lost to follow up to meet the set guidelines which led to adverse outcomes. But upon comparison, the LSCS rate was higher than the rates observed in many other studies, which might be because of the early induction of labour in comparison to others. In our study, none of the women had any post-partum complications, but in the study done by Ghiimrie SP et al., $11.5 \%$ of PPH was seen, $45 \%$ had meconium stained liquor. In a study done by Padmaja et al., the complications observed were lower and were in comparison with the present study findings. Thus, this finding gives us an implication upon the importance of properly timed antenatal visits and the importance of educating the women to approach the health care facility for properly timed labour to avoid complications at a later date.

The average Apgar score at 1 minute was 7 and at the end of 5 minutes, it was 8 . This finding was in consonance with the findings made by Padmaja et al. but in the study done by Ghimrie SP et al. 8 it was $<7$ at 1 minute. Around $50 \%$ of the children were admitted to ICU, which was way more than the findings made by the studies done by Ghimirie SP et al. ${ }^{8}$ Deevar et al. ${ }^{9}$ and Padmaja et al. ${ }^{7}$ where it was around $44 \%$. This step was taken to keep the children under observation to avoid uneventful circumstances.

This was found to be a good measure as the mortality among the neonates was higher in other studies when compared to the present study due to varied outcomes like the timing of diagnosis, timing of labour, mode of delivery and the interventions adopted. In the study conducted by Padmaja et al., ${ }^{7}$ they have opined that as the pathophysiology behind foetal distress is clear, no amount of antenatal visits can suffice or supplement the appropriate post-natal care that is provided to the infants, which has been proved in our study as the appropriate care that is provided by timely assessment and vigilant and meticulous care given in the NICU has helped in nil mortality in the neonates born.

\section{CONCLUSIONS}

Intrahepatic cholestasis of pregnancy is common among pregnant women which has an impact on feto-maternal outcomes. It was found to be responsible for a large number of perinatal and neonatal deaths especially after 36 weeks of gestation, but if appropriate antenatal care and timely intervention were implemented it would better the outcomes of pregnancy. A large prospective study is needed to establish the clinical and epidemiological pattern of this obstetric problem. Alongside, awareness must be increased among the health personnel caring for the pregnant women that pruritus of pregnancy is a high-risk condition and close foetal monitoring and timely intervention will decrease perinatal mortality significantly.

Data sharing statement provided by the authors is available with the full text of this article at jemds.com.

Financial or other competing interests: None.

Disclosure forms provided by the authors are available with the full text of this article at jemds.com. 


\section{REFERENCES}

[1] Williamson C, Geenes V. Intrahepatic cholestasis of pregnancy. Obstet Gynecol 2014;124(1):120-33.

[2] Geenes V, Williamson C, Chappell LC. Intrahepatic cholestasis of pregnancy. The Obstetrician \& Gynaecologist 2016;18(4):273-81.

[3] Madazli R, Yuksel MA, Oncul M, et al. Pregnancy outcomes and prognostic factors in patients with intrahepatic cholestasis of pregnancy. J Obstet Gynaecol 2015;35(4):358-61.

[4] Kenyon AP, Piercy CN, Girling J, et al. Obstetric cholestasis, outcome with active management: a series of 70 cases. BJOG 2002;109(3):282-8.

[5] Sharma A, Choudhary P, Acharya R, et al. Intrahepatic cholestasis of pregnancy: a serious but underestimated problem. J South Asian Feder Obst Gynae 2012;4(2):1189.

[6] Cardoso PM. A study of obstetric cholestasis in Goa Medical College. IOSR Journal of Dental and Medical Sciences (IOSR - JDMS) 2020;19(2):28-32.

[7] Padmaja M, Bhaskar P, Kumar GJ, et al. A study of obstetric cholestasis. J Obstet Gynecol India 2010;60(3):225-31.

[8] Ghimire SP, Ghimire A, Jha GS, et al. Feto-maternal outcomes in intrahepatic cholestasis in pregnancy in a tertiary care centre in Eastern Nepal. Journal of Nobel Medical College 2016;5(1):20-5.

[9] Deveer R, Engin-Ustun Y, Celen S, et al. Two-year experience of obstetric cholestasis: outcome and management. Clin Exp Obstet Gynecol 2011;38(3):256-9. 